\title{
Erythromycin infusion prior to endoscopy for acute nonvariceal upper gastrointestinal bleeding: a pilot randomized controlled trial
}

Hee Kyong $\mathrm{Na}^{1}$, Hwoon-Yong Jung ${ }^{1}$, Dong Woo Seo ${ }^{2}, \mathrm{Hyun} \mathrm{Lim}^{1}$, Ji Yong Ahn' ${ }^{1}$, Jeong Hoon Lee ${ }^{1}$, Do Hoon Kim ${ }^{1}$, Kee Don $\mathrm{Choi}^{1}$, Ho June Song ${ }^{1}$, Gin Hyug Lee ${ }^{1}$, and Jin-Ho Kim ${ }^{1}$

Departments of ${ }^{1}$ Gastroenterology and ${ }^{2}$ Emergency Medicine, Asan Medical Center, University of Ulsan College of Medicine, Seoul, Korea

Received: April 12, 2016

Revised : May 20, 2016

Accepted: May 30, 2016

\section{Correspondence to}

Hwoon-Yong Jung, M.D.

Department of Gastroenterology, Asan Medical Center, University of Ulsan College of Medicine, 88 Olympic-ro 43-gil, Songpa-gu, Seoul 05505, Korea

Tel: +82-2-3010-3197

Fax: +82-2-476-0824

E-mail: hyjung@amc.seoul.kr
Background/Aims: The aim of this study was to compare the effects of erythromycin infusion and gastric lavage in order to improve the quality of visualization during emergency upper endoscopy.

Methods: We performed a prospective randomized pilot study. Patients presented with hematemesis or melena within 12 hours and were randomly assigned to the erythromycin group (intravenous infusion of erythromycin), gastric lavage group (nasogastric tube placement with gastric lavage), or erythromycin + gastric lavage group (both erythromycin infusion and gastric lavage). The primary outcome was satisfactory visualization. Secondary outcomes included identification of a bleeding source, the success rate of hemostasis, duration of endoscopy, complications related to erythromycin infusion or gastric lavage, number of transfused blood units, rebleeding rate, and bleeding-related mortality.

Results: A total of 43 patients were randomly assigned: 14 patients in the erythromycin group; 15 patients in the gastric lavage group; and 14 patients in the erythromycin + gastric lavage group. Overall satisfactory visualization was achieved in $81 \%$ of patients: $92.8 \%$ in the erythromycin group; $60.0 \%$ in the gastric lavage group; and $92.9 \%$ in the erythromycin + gastric lavage group, respectively ( $p=$ 0.055). The identification of a bleeding source was possible in all cases. The success rate of hemostasis, duration of endoscopy, and number of transfused blood units did not significantly differ between groups. There were no complications. Rebleeding occurred in three patients (7.0\%). Bleeding-related mortality was not reported.

Conclusions: Intravenous erythromycin infusion prior to emergency endoscopy for acute nonvariceal upper gastrointestinal bleeding seems to provide satisfactory endoscopic visualization.

Keywords: Gastrointestinal hemorrhage; Erythromycin; Gastric lavage; Endoscopy

\section{INTRODUCTION}

Acute nonvariceal upper gastrointestinal bleeding (NVUGIB) is a commonly encountered medical emer- gency situation with a reported incidence that ranges from 50 to 150 cases per 100,000 adults per year [1-5]. NVUGIB carries a substantial mortality risk of $10 \%$. In NVUGIB patients, esophagogastroduodenoscopy (EGD) 
plays a key role when investigating hemorrhage and the treatment of bleeding foci. However, inadequate visualization during emergency endoscopy precludes the identification of the bleeding focus and performing endoscopic hemostasis.

Erythromycin (EM), a motilin receptor agonist, accelerates gastric emptying by inducing gastric antral contractions that are similar to phase III of the interdigestive migrating motor complex [6-8]. EM has been administered to patients with diabetic gastroparesis. Recently, randomized controlled studies have shown that the infusion of EM prior to endoscopy effectively improves visualization and decreases the need for repeat EGD in patients with upper gastrointestinal (GI) bleeding [9-11]. The American College of Gastroenterology (ACG) recommends the intravenous infusion of $250 \mathrm{mg}$ EM before emergent endoscopy for patients with upper GI bleeding, and gastric tube lavage is no longer recommended [12]. However, in many Asian countries, nasogastric tube placement and gastric lavage (GL) are routinely performed on patients presenting with melena or hematemesis. Furthermore, previously reported studies on the effects of administering EM prior to endoscopy in patients with upper GI bleeding were conducted in the United States and in European countries. Therefore, we performed our present pilot study to identify the effect of administering EM infusion in order to improve the quality of visualization during emergency endoscopy in Korean patients with NVUGIB.

\section{METHODS}

\section{Study population}

A prospective, randomized controlled trial was conducted. Patients $>20$ years of age who presented with hematemesis or melena within 12 hours and were referred to the Emergency Department at Asan Medical Center between December 2012 and August 2013 were included. A stratified randomization procedure was used wherein randomization was stratified as hematemesis and melena. Patients were excluded for the following criteria: (1) known or suspicious liver cirrhosis; (2) known allergy to EM or other macrolides; (3) corrected QT (QTc) interval $>0.45$ seconds on electrocardiography; (4) Glasgow Coma Scale score < 15; (5) systolic blood pressure < $90 \mathrm{mmHg}$ and pulse rate $>110$ beats/min despite fluid resuscitae tion; (6) the concomitant use of theophylline, warfarin, terfenadine, astemizole, carbamazepine, or cyclosporine because of possible interactions with EM; (7) prior gastrectomy; (8) hepatic dysfunction (aspartate transaminase or alanine transaminase $\geq 2 \times$ the normal upper limit); or (9) currently pregnant or lactating.

\section{Study protocols}

The eligible patients were randomly assigned to one of three groups: (1) the EM group (intravenous infusion of EM); (2) GL group (nasogastric tube placement with GL); or (3) EM + GL group (both EM infusion and GL). For EM infusion, $250 \mathrm{mg}$ EM was mixed with $50 \mathrm{~mL}$ isotonic saline solution and intravenously administered over 30 minutes. Emergency endoscopy was performed 20 to 60 minutes after EM infusion. For GL, a 16-French nasogastric tube was positioned in the stomach and GL with 1 $\mathrm{L}$ of tepid water was performed. GL was repeated every hour, and the endoscopic exam was performed within 30 minutes after the last gastric irrigation. All adverse effects were recorded during and after infusion or GL. All patients were underwent emergency endoscopy within 4 hours after arrival at the emergency department. An endoscopist, who was unaware of the assignment group, evaluated the quality of visualization during EGD and performed endoscopic hemostasis if the bleeding focus was identified. The method of hemostasis was chosen by the performing endoscopist. The patients who were treated with endoscopic hemostasis received an intravenous proton pump inhibitor for 72 hours.

The decision to admit the patient or not was made based on clinical implications. The results of the laboratory tests (white blood cell count, hemoglobin level, platelet count, prothrombin time, and blood urea nitrogen), the number of transfused blood units, instances of rebleeding, and mortality ratings on days 7 and 30 were recorded. The Glasgow-Blatchford score and pre-endoscopic and post-endoscopic Rockall score were calculated. The discharged patients who failed to attend their follow-up visit were contacted by telephone. The current study protocol was approved by the Institutional Review Board of the Asan Medical Center (No. 2012-0696). This clinical study was also approved by the Korean Food and Drug Administration (No. S2012-1361-0002). Written informed consent was obtained from each patient at the 


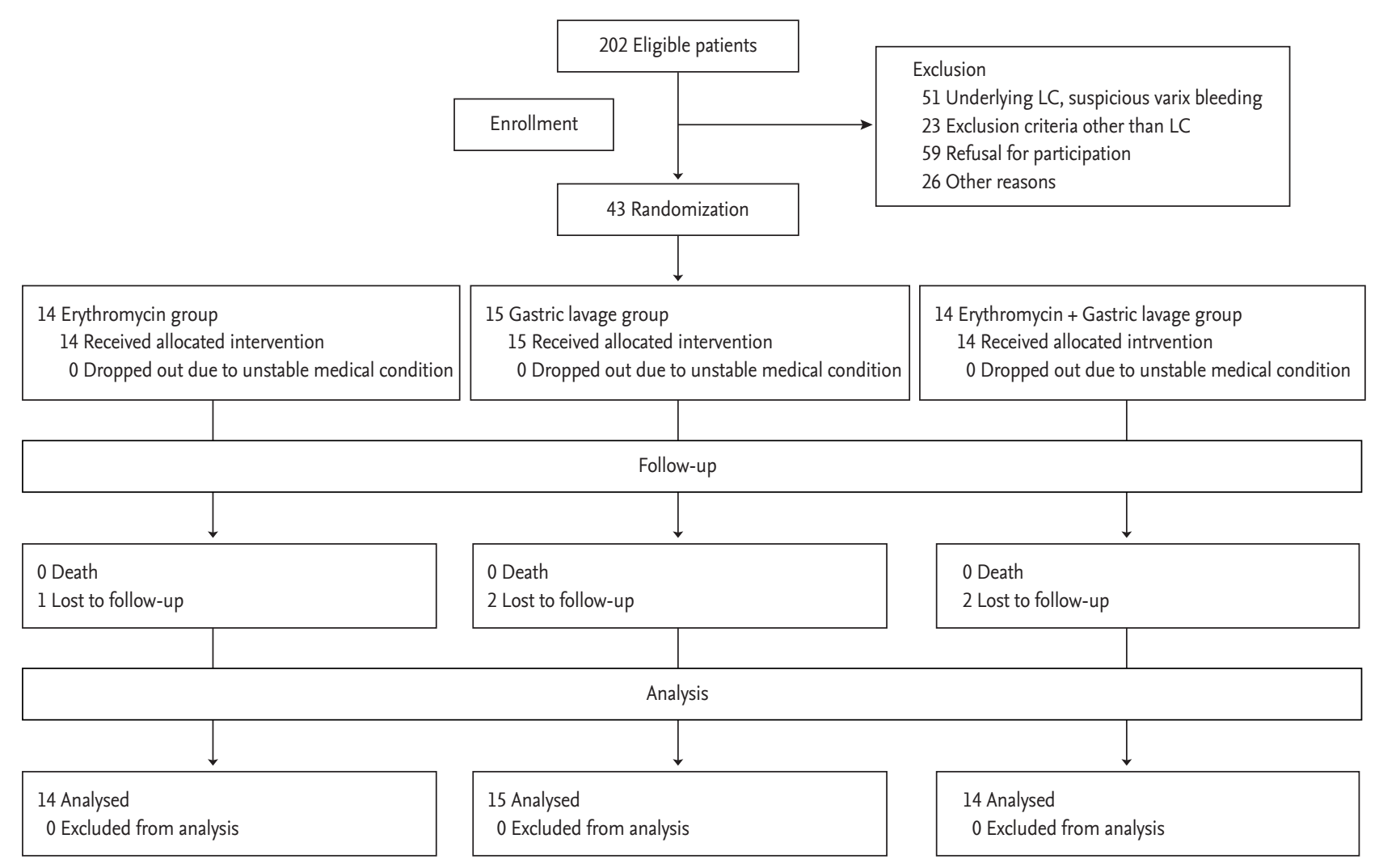

Figure 1. Flow chart of the study population. LC, liver cirrhosis.

time of enrollment.

\section{End points}

The primary end point was the quality of GI visualization according the scoring system used by Frossard et al. [11]. An endoscopist scored each of the four areas of stomach and duodenum (fundus, corpus, antrum, and bulb) from o to 2 (o, $<25 \%$ of the surface was visible; 1 , $25 \%$ to $75 \%$ visible; $2,>75 \%$ visible). The total score was the sum of the scores for each of these four areas. Therefore, the score ranged from o to 8 . A score $\geq 6$ was considered satisfactory visualization, while $\leq 5$ was considered unsatisfactory visualization. The secondary end points included the ability to identify the bleeding source, the success rate of hemostasis, duration of the emergency endoscopy, adverse effects related to EM infusion or GL, number of transfused blood units, rebleeding rate, and bleeding-related mortality.

\section{Statistical analysis}

The categorical variables are expressed as percentag- es and were compared between groups using the chisquare test and Fisher exact test. The quantitative variables are expressed as the mean \pm standard deviation. Analysis of variance was conducted to compare the numerical variables between the three groups. Data that were not normally distributed were compared among groups using the Kruskal-Wallis test. Analyses were performed on an intention to treat basis and included all patients who underwent randomization. Statistical significance was established at $p<0.05$. All statistical analyses were conducted using SPSS version 18.0 (SPSS Inc., Chicago, IL, USA).

\section{RESULTS}

Fig. 1 shows the flow of the study patients. A total 202 patients were assessed for eligibility between December 2012 and August 2013. Of these patients, 43 patients were included in the study and randomized as follows: 14 patients were randomly assigned to receive EM alone 
Table 1. Baseline clinical and biological characteristics of the study patients

\begin{tabular}{|c|c|c|c|c|}
\hline Characteristic & EM group $(n=14)$ & GL group $(n=15)$ & $\mathrm{EM}+\mathrm{GL}$ group $(\mathrm{n}=14)$ & $p$ value \\
\hline Age, yr & $60 \pm 14$ & $63 \pm 12$ & $57 \pm 15$ & 0.499 \\
\hline Sex, male/female & $12 / 2$ & $13 / 2$ & $13 / 1$ & 1.000 \\
\hline \multicolumn{5}{|l|}{ Presenting symptom } \\
\hline Hematemesis & $5(35 \cdot 7)$ & $5(33 \cdot 3)$ & $5(35 \cdot 7)$ & 1.000 \\
\hline Melena & $9(64.3)$ & $10(66.7)$ & $9(64.3)$ & 1.000 \\
\hline Onset time (hours prior) & $14(2-24)$ & $7(1-72)$ & $17(2.8-72)$ & 0.846 \\
\hline \multicolumn{5}{|l|}{ Comorbidities } \\
\hline Renal failure & 0 & $3(20.0)$ & 0 & 0.096 \\
\hline Malignancy & $1(7.1)$ & $4(26.7)$ & $1(7.1)$ & 0.329 \\
\hline CVA & $2(14.3)$ & 0 & $1(7.1)$ & 0.302 \\
\hline IHD & $2(14 \cdot 3)$ & $3(20.0)$ & o & 0.342 \\
\hline \multicolumn{5}{|l|}{ Premedication } \\
\hline Aspirin & $2(14.3)$ & $3(20.0)$ & $2(14 \cdot 3)$ & 1.000 \\
\hline Antiplatelet & $2(14.3)$ & $3(20.0)$ & $2(14.3)$ & 1.000 \\
\hline NSAIDs & $1(7 \cdot 1)$ & o & $1(7.1)$ & 0.535 \\
\hline PPI & $1(7.1)$ & 0 & 0 & 0.651 \\
\hline Steroids & o & $2(13 \cdot 3)$ & o & 0.318 \\
\hline History of peptic ulcer & $1(7.1)$ & $1(6.7)$ & $3(21.4)$ & 0.500 \\
\hline History of GI bleeding & $1(7.1)$ & 0 & $3(21.4)$ & 0.110 \\
\hline \multicolumn{5}{|l|}{ Vital sign } \\
\hline $\mathrm{SBP}, \mathrm{mmHg}$ & $120 \pm 24$ & $120 \pm 22$ & $120 \pm 23$ & 0.996 \\
\hline $\mathrm{DBP}, \mathrm{mmHg}$ & $76 \pm 14$ & $75 \pm 14$ & $78 \pm 21$ & 0.990 \\
\hline $\mathrm{PR}, / \mathrm{min}$ & $89 \pm 14$ & $88 \pm 21$ & $96 \pm 25$ & 0.588 \\
\hline \multicolumn{5}{|l|}{ Laboratory findings } \\
\hline Hemoglobin, g/dL & $11.0 \pm 3.0$ & $9.5 \pm 3.2$ & $11.6 \pm 3.4$ & 0.259 \\
\hline $\mathrm{WBC}, / \mathrm{mm}^{3}$ & $8.3 \pm 3.3$ & $9.5 \pm 3.0$ & $8.5 \pm 3.0$ & 0.521 \\
\hline Platelet, $\times 10^{3} / \mathrm{mm}^{3}$ & $213 \pm 63$ & $233 \pm 108$ & $251 \pm 45$ & 0.185 \\
\hline $\mathrm{PT}, \%$ & $95 \pm 10$ & $88 \pm 14$ & $106 \pm 12$ & $<0.001$ \\
\hline Urea, mmol/L & $30 \pm 15$ & $40 \pm 29$ & $25 \pm 13$ & 0.479 \\
\hline Glasgow-Blatchford score & $7(4-11)$ & $9\left(5^{-12}\right)$ & $6(3-12)$ & 0.527 \\
\hline Pre-endoscopic Rockall score & $1(1-3)$ & $2(1-4)$ & $1(0-1)$ & 0.052 \\
\hline Post-endoscopic Rockall score & $4(4-5)$ & $5(4-6)$ & $3 \cdot 5(2-4)$ & 0.134 \\
\hline Positive Helicobacter pylori test & $4(28.6)$ & $4(26.7)$ & $4(28.6)$ & 0.514 \\
\hline
\end{tabular}

Values are presented as mean $\pm \mathrm{SD}$, number (\%), or median (interquartile range).

EM, erythromycin; GL, gastric lavage; CVA, cerebrovascular accident; IHD, ischemic heart disease; NSAID, nonsteroidal anti-inflammatory drug; PPI, proton pump inhibitor; GI, gastrointestinal; SBP, systolic blood pressure; DBP, diastolic blood pressure; PR, pulse rate; WBC, white blood cell; PT, prothrombin time.

before EGD; 15 patients were assigned to receive GL; and 14 patients were assigned to receive both EM and GL. All patients received the allocated interventions. Among these patients, 41 patients were followed for 7 days, and
38 patients were followed for 30 days.

Table 1 lists the baseline characteristics of the study population. The groups were well matched in terms of age, sex, and presenting symptoms. The onset time of 
Table 2. Endoscopic features and treatments used in the study series

\begin{tabular}{|c|c|c|c|c|}
\hline Variable & EM group $(n=14)$ & GL group $(n=15)$ & $\mathrm{EM}+\mathrm{GL} \operatorname{group}(\mathrm{n}=14)$ & $p$ value \\
\hline Identification of bleeding focus & $14(100)$ & $15(100)$ & $14(100)$ & \\
\hline Duration of endoscopy, min & $11 \pm 4$ & $11 \pm 8$ & $14 \pm 10$ & 0.743 \\
\hline Endoscopic findings & & & & 0.729 \\
\hline Gastric ulcer & $3(21.4)$ & $6(40.0)$ & $3(21.4)$ & \\
\hline Duodenal ulcer & $1(7.1)$ & $1(6.7)$ & $2(14 \cdot 3)$ & \\
\hline Mallory-Weiss tear & $2(14 \cdot 3)$ & o & $2(14 \cdot 3)$ & \\
\hline Dieulafoy lesion & o & $1(6.7)$ & O & \\
\hline Malignancy & $3(21.4)$ & $2(13 \cdot 3)$ & $2(14 \cdot 3)$ & \\
\hline Post-ESD ulcer & $4(28.6)$ & $1(6.7)$ & $2(14 \cdot 3)$ & \\
\hline Others & 0 & $2(13 \cdot 3)$ & $1(7.1)$ & \\
\hline No evidence of bleeding & $1(7 \cdot 1)$ & $2(13 \cdot 3)$ & $2(14 \cdot 3)$ & \\
\hline Hemostatic treatment & $7(50.0)$ & $7(46.7)$ & $9(64 \cdot 3)$ & 0.603 \\
\hline Hemostatic method & & & & 0.938 \\
\hline Hemoclip & $1(14 \cdot 3)$ & 0 & $1(11.1)$ & \\
\hline Thermal coagulation & $2(28.6)$ & $2(28.6)$ & $3(33 \cdot 3)$ & \\
\hline Fibrin glue & $1(14 \cdot 3)$ & 0 & $1(11.1)$ & \\
\hline Epinephrine injection \pm spraying & o & o & $1(11.1)$ & \\
\hline Multimodal & $3(42.9)$ & $5(71.4)$ & $3(33 \cdot 3)$ & \\
\hline
\end{tabular}

Values are presented as number (\%) or mean \pm SD.

EM, erythromycin; GL, gastric lavage; ESD, endoscopic submucosal dissection.

Table 3. Primary and secondary end points

\begin{tabular}{|c|c|c|c|c|}
\hline Variable & EM group $(n=14)$ & GL group $(n=15)$ & $\mathrm{EM}+\mathrm{GL} \operatorname{group}(\mathrm{n}=14)$ & $p$ value \\
\hline \multicolumn{5}{|l|}{ Primary endpoint } \\
\hline Visualization score & $8(6-8)$ & $7(5-8)$ & $8(8)$ & 0.082 \\
\hline Satisfactory visualization $(\geq 6)$ & $13(92.9)$ & $9(60.0)$ & $13(92.9)$ & 0.055 \\
\hline \multicolumn{5}{|l|}{ Secondary end point } \\
\hline Identification of the source & $14(100)$ & $15(100)$ & $14(100)$ & \\
\hline Success rate of hemostasis & $7 / 7(100)$ & $6 / 7(85 \cdot 7)$ & $9 / 9(100)$ & 0.609 \\
\hline Adverse event & o & o & o & \\
\hline No. of transfused RBCs & $1(0-2)$ & $2(0-3)$ & $2(0-3)$ & 0.391 \\
\hline Rebleeding & $1(7 \cdot 1)$ & $1(6.7)$ & $1(7 \cdot 1)$ & 1.000 \\
\hline Death & o & o & o & \\
\hline
\end{tabular}

Values are presented as median (interquartile range) or number (\%).

EM, erythromycin; GL, gastric lavage; RBC, red blood cell.

bleeding, comorbidities, premedication, initial systolic blood pressure, initial diastolic blood pressure and pulse pressure, mean hemoglobin, and mean platelet counts did not significantly different between the three groups. Even though there was a significant difference between the three groups with regard to the mean pro- thrombin time $(95 \% \pm 10 \%$ in EM group, $88 \% \pm 14 \%$ in GL group, and $106 \% \pm 12 \%$ in the EM + GL group, $p<$ 0.001 ), all values are within the normal range. For the patients in the GL group and in EM + GL groups, the mean number of performed GL was 1.4 (range, 1 to 3 ).

The endoscopic features and treatments in each group 


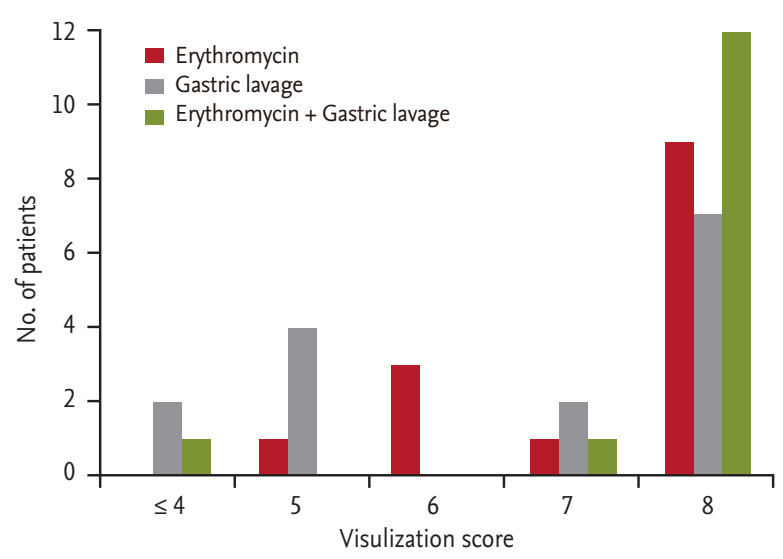

Figure 2. Visualization score at emergency endoscopy.

are presented in Table 2. The identification of the bleeding focus was possible in all patients. The mean duration of the endoscopic procedure, endoscopic findings, hemostatic treatments, and hemostatic methods did not significantly differ between the groups.

Table 3 indicates the primary and secondary end points. No statistical difference in terms of the median visualization score (interquartile range) was found between the three study groups. Although the percentage of patients with a satisfactory visualization score did not differ significantly between these three groups, it tended to be lower in the GL group. Each of the visualization scores of the three groups are shown in Fig. 2. The success rate of hemostasis was 100\% (seven of seven patients) in the EM group, $85.7 \%$ (six of seven patients) in the GL group, and 100\% (nine of nine patients) in EM + GL group. One patient in the GL group demonstrated spurting bleeding from the gastric ulcer base, and endoscopic hemostasis failed. He additionally underwent emergency angiographic embolization and was successfully treated. There were no complications associated with EM infusion or GL. Rebleeding occurred in three patients (one patient in each group). Bleeding-related mortality was not reported.

\section{DISCUSSION}

Our current study findings show that intravenous EM infusion may be of help to improve visualization during emergency endoscopy. Even though there were no statistically significant differences found between our three study groups, the percentage of satisfactory visualization in the EM infusion group was high at $92.9 \%$, and that in GL group was low at $60.0 \%$. Furthermore, additive GL after EM infusion did not show a clinical benefit over EM infusion alone. This implies that intravenous EM infusion alone can be effective enough to obtain satisfactory endoscopic view. A prospective multicenter randomized controlled trial in France reported results that are consistent with our current findings. When 253 patients with upper GI bleeding were randomly assigned to the EM infusion group without a nasogastric tube, nasogastric tube placement without EM, or intravenous EM infusion combined with nasogastric tube placement, overall satisfactory visualization was achieved in $85 \%$ of patients and the between-group differences were not significant [13]. Additionally, as the results of our current analyses show, the mean number of blood units transfused, the rebleeding rate, and the mortality rate did not significantly differ between the study groups. Several prospective randomized trials have reported the benefit of EM over treatment without EM infusion in patients with acute upper GI bleeding [9-11]. Satisfactory visualizations were achieved $65 \%$ to $90 \%$ of the patients who were treated with intravenous EM injection in those previous trials. In our present study, the percentage of patients who achieved satisfactory visualization was as high as $92.9 \%$. The reason for this is likely that we excluded patients with unstable vital signs (systolic blood pressure $<90 \mathrm{mmHg}$ and pulse rate $>110$ beats/ min) despite fluid resuscitation as these cases had the possibility of massive bleeding into the stomach.

Previously reported randomized controlled trials on the effects of EM prior to endoscopy in patients with upper GI bleeding were conducted in the United States and in some European countries [9-11,13,14]. Therefore, the guidelines for patients with upper GI bleeding in these countries recommend EM treatment prior to an emergency endoscopy. In 2012, ACG first recommended the intravenous infusion of $250 \mathrm{mg}$ EM before emergent endoscopy for patients with upper GI bleeding [12]. The European Society of Gastrointestinal Endoscopy guidelines also recommend intravenous EM for patients with clinically severe or ongoing, active upper GI bleeding [15]. However, international consensus recommendations regarding the management of patients with NVUGIB do not recommend the routine usage of proki- 
netic agents before endoscopy in order to increase the diagnostic yield [16]. The Asian Pacific Working Group consensus on NVUGIB did not refer to the use of prokinetics, including EM, before emergency endoscopy [17]. Notably, nasogastric tube insertion and GL with normal saline are usually performed in many Asian countries to check for active bleeding and clear the stomach. Therefore, our current study is clinically significant in that it demonstrates the beneficial effects of EM treatment before emergency endoscopy in a Korean cohort.

In our current study series, we found no adverse events related to EM infusion. Allergic drug reactions to macrolides are known to be extremely rare [18]. As one of the most severe adverse events, QTc-interval prolongation is only likely to be a potential problem in patients with heart disease or other factors, or patients who are receiving agents that may further delay ventricular repolarization [19]. Drug interactions such as EM-induced digoxin toxicity are reported to occur when EM is repeatedly administrated $[20,21]$. No adverse events related to the use of EM before emergency endoscopy were reported in previous studies [9-11]. Hence, if high-risk patients are properly excluded, EM infusion can be safely used. Furthermore, EM infusion is a cost-effective treatment. In a previous study on the cost-effectiveness of EM infusion before EGD for patients with acute upper GI bleeding, EM prior to EGD resulted in a cost saving of US\$486 and 0.00007 quality-adjusted life-years [22].

Our study had some limitations of note. First, we did not include patients with severe comorbidities or unstable vital signs, and this prevented conclusions about significant mortality-related differences between our study groups. Second, our patients underwent emergency endoscopy within 4 hours after arrival at the emergency department, and it is difficult to conduct an emergency endoscopy in clinical settings. However, our present analyses indicated beneficial effects of EM infusion before emergency endoscopy in a Korea population with well-defined end points of visualization.

In conclusion, intravenous EM infusion prior to emergency endoscopy for acute NVUGIB may be of help to provide satisfactory endoscopic visualization. Considering the potential adverse effects of clearing the stomach and the good safety profile of EM, nasogastric tube placement and GL can be replaced by EM infusion in patients with NVUGB.

\section{KEY MESSAGE}

1. Erythromycin accelerates gastric emptying by inducing gastric antral contraction.

2. Intravenous erythromycin infusion prior to emergency endoscopy for acute nonvariceal upper gastrointestinal bleeding seems to provide satisfactory endoscopic visualization in Korean patients.

\section{Conflict of interest}

No potential conflict of interest relevant to this article was reported.

\section{REFERENCES}

1. Targownik LE, Nabalamba A. Trends in management and outcomes of acute nonvariceal upper gastrointestinal bleeding: 1993-2003. Clin Gastroenterol Hepatol 2006;4:1459-1466.

2. van Leerdam ME, Vreeburg EM, Rauws EA, et al. Acute upper GI bleeding: did anything change? Time trend analysis of incidence and outcome of acute upper GI bleeding between 1993/1994 and 2000. Am J Gastroenterol 2003;98:1494-1499.

3. Lassen A, Hallas J, Schaffalitzky de Muckadell OB. Complicated and uncomplicated peptic ulcers in a Danish county 1993-2002: a population-based cohort study. Am J Gastroenterol 2006;101:945-953.

4. Zhao Y, Encinosa W. Hospitalizations for gastrointestinal bleeding in 1998 and 2006: statistical brief \#65. In: Healthcare Cost and Utilization Project, ed. Healthcare Cost and Utilization Project (HCUP) Statistical Briefs. Rockville: Agency for Healthcare Research and Quality (US), 2006 [cited 2017 Jan 23]. Available from: https://www. ncbi.nlm.nih.gov/books/NBK54562/.

5. Theocharis GJ, Thomopoulos KC, Sakellaropoulos G, Katsakoulis E, Nikolopoulou V. Changing trends in the epidemiology and clinical outcome of acute upper gastrointestinal bleeding in a defined geographical area in Greece. J Clin Gastroenterol 2008;42:128-133.

6. Weber FH Jr, Richards RD, McCallum RW. Erythromycin: a motilin agonist and gastrointestinal prokinetic agent. Am J Gastroenterol 1993;88:485-490. 
7. Lin HC, Sanders SL, Gu YG, Doty JE. Erythromycin accelerates solid emptying at the expense of gastric sieving. Dig Dis Sci 1994;39:124-128.

8. Janssens J, Peeters TL, Vantrappen G, et al. Improvement of gastric emptying in diabetic gastroparesis by erythromycin: preliminary studies. N Engl J Med 1990;322:10281031.

9. Carbonell N, Pauwels A, Serfaty L, Boelle PY, Becquemont L, Poupon R. Erythromycin infusion prior to endoscopy for acute upper gastrointestinal bleeding: a randomized, controlled, double-blind trial. Am J Gastroenterol 2006;101:1211-1215.

10. Coffin B, Pocard M, Panis Y, et al. Erythromycin improves the quality of EGD in patients with acute upper GI bleeding: a randomized controlled study. Gastrointest Endosc 2002;56:174-179.

11. Frossard JL, Spahr L, Queneau PE, et al. Erythromycin intravenous bolus infusion in acute upper gastrointestinal bleeding: a randomized, controlled, double-blind trial. Gastroenterology 2002;123:17-23.

12. Laine L, Jensen DM. Management of patients with ulcer bleeding. Am J Gastroenterol 2012;107:345-36o.

13. Pateron D, Vicaut E, Debuc E, et al. Erythromycin infusion or gastric lavage for upper gastrointestinal bleeding: a multicenter randomized controlled trial. Ann Emerg Med 2011;57:582-589.

14. Rudzki S, Czekalowski S, Michalak K, Kusz W, Fularz A.
Erythromycin improves quality of endoscopy for acute upper gastrointestinal bleeding. Wiad Lek 2006;59:490491.

15. Gralnek IM, Dumonceau JM, Kuipers EJ, et al. Diagnosis and management of nonvariceal upper gastrointestinal hemorrhage: European Society of Gastrointestinal Endoscopy (ESGE) Guideline. Endoscopy 2015;47:a1-a46.

16. Barkun AN, Bardou M, Kuipers EJ, et al. International consensus recommendations on the management of patients with nonvariceal upper gastrointestinal bleeding. Ann Intern Med 2010;152:101-113.

17. Sung JJ, Chan FK, Chen M, et al. Asia-Pacific Working Group consensus on non-variceal upper gastrointestinal bleeding. Gut 2011;60:1170-1177.

18. Rubinstein E. Comparative safety of the different macrolides. Int J Antimicrob Agents 2001;18 Suppl 1:S71-S76.

19. Oberg KC, Bauman JL. QT interval prolongation and torsades de pointes due to erythromycin lactobionate. Pharmacotherapy 1995;15:687-692.

20. Morton MR, Cooper JW. Erythromycin-induced digoxin toxicity. DICP 1989;23:668-670.

21. Friedman HS, Bonventre MV. Erythromycin-induced digoxin toxicity. Chest 1982;82:202.

22. Winstead NS, Wilcox CM. Erythromycin prior to endoscopy for acute upper gastrointestinal haemorrhage: a cost-effectiveness analysis. Aliment Pharmacol Ther 2007;26:1371-1377. 